\title{
Studies of laser-driven radiative blast waves
}

M. J. Edwards, J.F. Hansen, A.D. Edens, T. Ditmire, R.G. Adams, P. Rambo, L. Ruggles, I.C. Smith, J.L. Porter

April 30, 2004

High Energy Density Laboratory Astrophysics (HEDLA) Tucson, AZ, United States

May 10, 2004 through May 13, 2004 
This document was prepared as an account of work sponsored by an agency of the United States Government. Neither the United States Government nor the University of California nor any of their employees, makes any warranty, express or implied, or assumes any legal liability or responsibility for the accuracy, completeness, or usefulness of any information, apparatus, product, or process disclosed, or represents that its use would not infringe privately owned rights. Reference herein to any specific commercial product, process, or service by trade name, trademark, manufacturer, or otherwise, does not necessarily constitute or imply its endorsement, recommendation, or favoring by the United States Government or the University of California. The views and opinions of authors expressed herein do not necessarily state or reflect those of the United States Government or the University of California, and shall not be used for advertising or product endorsement purposes. 


\title{
Studies of laser-driven radiative blast waves
}

\author{
A. D. Edens and T. Ditmire \\ Department of Physics, University of Texas at Austin, Austin, TX 78712
}

J. F. Hansen and M. J. Edwards

Lawrence Livermore National Laboratory, Livermore, CA, 94550

R.G. Adams, P. Rambo, L. Ruggles, I.C. Smith, and J. L. Porter

Sandia National Laboratories, Albuquerque, NM, 87059

\begin{abstract}
We have performed two sets of experiments looking at laser-driven radiating blast waves. In one set of experiments the effect of a drive laser's passage through a background gas on the hydrodynamical evolution of blast waves was examined. It was found that the laser's passage heats a channel in the gas, creating a region where a portion of the blast wave front had an increased velocity, leading to the formation of a bump-like protrusion on the blast wave. The second set of experiments involved the use of regularly spaced wire arrays to induce perturbations on a blast wave surface. The decay of these perturbations as a function of time was measured for various wave number perturbations and found to be in good agreement with theoretical predictions.
\end{abstract}


Physicists and astronomers have long been studying the stability of shock waves ${ }^{1-}$

4. In particular, there are several instabilities of interest associated with the formation and evolution of shock waves produced by supernovae, i.e. supernova remnants(SNRs) ${ }^{2,5}$. One important hydrodynamic feature associated with some radiative SNRs, an overstability proposed by Vishniac et al. about 20 years ago, arises from a mismatch between the ram and thermal pressures at the surface of a blast wave which can cause a growing, oscillating ripple on that surface $\mathrm{e}^{3,6,7}$. Radiative SNR shocks are cooler and more compressible than non-radiative SNR shocks, leading to a thinner blast front which is susceptible to this Vishniac overstability ${ }^{8}$. The Vishniac overstability may be responsible for the large scale structure seen in some radiative SNRs, and may also play a role in the formation of stars ${ }^{4,9}$.

Vishniac et al. made quantitative predictions for the temporal evolution of small perturbations on a blast wave front and derived the growth rate of the overstability as a function of the effective adiabatic index of the shocked material and wave number of the oscillation $^{7}$. There have since been several attempts to confirm these growth rates both through simulation and experiment. The appearance of the Vishniac overstability in both 1-D and 2-D astrophysical simulations was seen by Blondin et $\mathrm{al}^{10}$, who observed perturbations on the order of $10 \%$ of the blast wave radius growing from a $1 \%$ density perturbation seed using the numerical hydrodynamics code VH-1. MacLow and Norman ran simulations ${ }^{11}$ using the numerical gas dynamics code ZEUS-2D which confirmed the theoretical growth rates of Vishniac et al. assuming the amplitude of the ripple was small compared to the blast waves' radius. 
Most experimental attempts to see the Vishniac overstability have used laser irradiation of a target to create a blast wave in a background gas ${ }^{12,13}$. These experiments take advantage of a laser's ability to deliver a large amount of energy to a small focal spot in a time span short compared to the evolution of the resulting explosion. There are several complications associated with these experiments that make it difficult to measure the growth rate of the overstability. The first complication for laser experiments in spherical geometry ${ }^{13}$, as opposed to cylindrical geometry ${ }^{12}$, is the effect of the laser's passage on the background gas, which can pre-ionize the gas. A second complication to experimental observation is that if the overstability grows from noise it is difficult to achieve large growth in the limited lifetime of the experiments. In this paper we describe experiments where we have looked at both of these issues and explored the evolution of perturbations by inducing ripples on a blast wave using regularly spaced wire arrays.

Some of the data described here was acquired on the Z-Beamlet ${ }^{14}$ laser at Sandia National Laboratories, which fired 500J-1000J, 1ns pulses of $1054 \mathrm{~nm}$ wavelength. The remainder of the data was taken on the Janus laser ${ }^{15}$ at Lawrence Livermore National Laboratory, which fired $10 \mathrm{~J}-150 \mathrm{~J}, 1 \mathrm{~ns}$ laser pulses at $527 \mathrm{~nm}$ wavelength. These pulses illuminated 500 micron diameter cylindrical solid targets immersed in 5-10 Torr of nitrogen or xenon gas. The resulting explosions created the blast waves that we studied. The setup used is diagramed in Figure 1. The inset in Figure 1 shows the main diagnostic employed: a dark-field telescope. This diagnostic is used in conjunction with a probe laser and is sensitive to density gradients, such as those that occur at the edge of a blast wave. The probe laser used with Z-Beamlet fired $\sim 80 \mathrm{~mJ}, 150 \mathrm{ps}$ pulses at $1064 \mathrm{~nm}$. The probe laser used with Janus fired $\sim 10 \mathrm{~mJ}, 2 \mathrm{~ns}$ pulses at $527 \mathrm{~nm}$. These lasers could be 
fired at a variable interval after the drive laser allowing us to examine the evolution of our blast wave over several microseconds. For part of the experiments, regularly spaced wire arrays were used to induce single frequency perturbations on blast waves. The wire array contained a square of open space approximately $3 \mathrm{~cm}$ on a side through which 30 gauge tin-copper wire was strung. The wires were spaced 2,4 , or $6 \mathrm{~mm}$ apart, corresponding to induced perturbations with spherical_numbers of $\sim 28,14$, and 9 given the size of the blast wave at the time it intersected the array.

The first experiments were performed without the wire array and looked at the evolution of the radius of the blast wave as a function of time. The position of any feature of a blast wave, such as the blast wave front, will evolve as $R(t)=\beta t^{\alpha}$. Blast waves that do not lose or gain a significant fraction of their initial energy during their evolution, i.e. those where radiation does not play an important role in the hydrodynamics, follow the Taylor-Sedov solution, where $\alpha=0.4{ }^{16}$. In blast waves where radiation is important, a solution with a lower $\alpha$ will be followed, as the energy loss via radiation causes these blast waves to slow down more quickly. Therefore the trajectory of a blast wave can show the importance of radiation on its hydrodynamics.

Images of blast waves produced by $1000 \mathrm{~J}$ laser pulses at various times along with a measured trajectory are shown in Figure 2 with 10 Torr of nitrogen as the background gas and in Figure 3 with 10 Torr of xenon as the background gas. The trajectory in nitrogen follows a $\mathrm{t}^{0.4}$ trajectory to a high degree of accuracy over its entire history. In contrast, while xenon at late times ( $>400 \mathrm{~ns})$ may expand as $\mathrm{t}^{0.4}$, at earlier times the trajectory appears to exhibit a lower $\alpha$. This lower exponent most likely arises from the fact that energy losses via radiation have an effect on the hydrodynamics of the blast 
wave. Another indication that radiation plays a much larger role for blast waves in xenon than in those traveling through nitrogen is the radiative precursor. When a blast wave radiates, some of the energy is absorbed by the background gas, creating a heated, ionized region in front of the blast wave. This ionized region shows up as a bright area on the dark field images. In Figure 2, while there is a noticeable glow surrounding the blast waves it is localized to the region immediately surrounding the blast wave, never extending more than $7 \mathrm{~mm}$ away from the edge of the blast wave. This can be contrasted with Figure 3, where the precursor region extends off the field of view, at least several $\mathrm{cm}$, demonstrating a significantly greater amount of radiation emitted from blast waves in xenon.

Another feature of note from these images is the presence of a "bump" feature on the laser side of the blast waves, especially those in xenon gas. A closer look at an example of this feature can be seen in Figure 4. A qualitatively similar feature occurs for blast waves in xenon at all drive laser energies from $10 \mathrm{~J}-1000 \mathrm{~J}$ and always occurs along the path of the laser focal cone. This feature arises from the interaction of the laser with the background gas. As the laser passes through the background gas, it heats and ionizes the gas it passes through. This creates a warm, lower density channel of gas where the blast wave will travel faster than in other regions, which results in the bump like feature seen in the data. Simulations on this phenomenon were performed using CALE, the Lawrence Livermore National Laboratory 2-D arbitrary Lagrangian Eulerian (ALE) code with a tabular equation of state (EOS) and interface tracking ${ }^{17}$. They indicate that for a $100 \mathrm{~J}$ drive laser pulse, the laser channel region is heated to up to $12 \mathrm{eV}$, comparable to what we expect the temperature of the shock heated gas to be. 
For the next set of experiments we placed wire arrays in the path of blast waves produced by $1000 \mathrm{~J}$ laser pulses in order to induce perturbations. When we did this for blast waves traveling through xenon, the radiation from the blast wave ablated the wire array, creating secondary blast waves that interfered with our main blast wave until they were outside our field of view. The formation of these secondary blast waves is illustrated in Figure 5. For blast waves traveling through nitrogen, we expected that the induced perturbations would decay away over time. Because radiation does not appear to play an important role in the energy dynamics of these blast waves, their blast fronts should be too thick for growth of the Vishniac overstability to occur. However, the radiative precursor around the nitrogen blast wave images in Figure 2 indicates that some radiation is emitted, which could lower the effective adiabatic index somewhat, resulting in slightly thinner blast waves. The adiabatic index for a perfect monatomic gas is 1.66 and we would expect that our adiabatic index would be somewhere between this value and 1.2, the value where growth begins to occur for the Vishniac overstability. Some of the images corresponding to each of our wire arrays are shown in Figure 6. In each case the induced perturbations clearly damp out, matching our intuition.

In order to measure the rate of this decay, we traced out the edge of the blast waves in polar coordinates. We Fourier transformed this curve to select the frequency corresponding to our wire array wave number. An example of the results of this procedure is shown in Figure 7. After we determined the amplitude of the frequency component of interest to us, we plotted this amplitude relative to the radius of the blast wave as a function of time and fit this data with a function of the form $\mathrm{R} / \mathrm{A}=\mathrm{C}^{*} \mathrm{t}^{\mathrm{p}}$. The results of this for the $6 \mathrm{~mm}$ array are shown in Figure 8. We compared these results to 
theoretical predictions for $\mathrm{p}$ as a function of spherical wave number _ from Vishniac et $\mathrm{al}^{7}$. This comparison is illustrated in Figure 9. Here we see the experimental data compared to theoretical predictions for various adiabatic indices. The best agreement occurs for an effective adiabatic index of 1.4-1.5. This agrees well with our intuition for the nitrogen gas in our experiment. In order to better determine the adiabatic index of our gas, we are currently performing simulations using the Hyades code ${ }^{18}$, a 1-D Lagrangian hydrodynamics code with tabular equation of state.

We have performed experiments designed to look at two complications that arise in the study of laser-produced blast wave studies of the Vishniac overstability. The first is the effect of the laser passage on the background gas in the experiment. We find that the passage of the drive laser beam in these experiments can create a warm, low density channel of gas that creates a region where the blast wave moves faster relative to the rest of the blast wave surface, creating an artificial bump-like feature. The second set of experiments have induced perturbations on blast waves and measured the resulting decay rate. We have compared this result to theoretical predictions relevant to the Vishniac overstability $^{7}$ and found good agreement. These results suggest that there are a wealth of physical processes occuring in laser-driven blast wave experiments. These processes can have unanticipated consequences, as illustrated by the formation of secondary blast waves on our wire array, produced by radiation from the primary blast wave in xenon. This work was performed under the auspices of the U.S. Department of Energy by the University of California, Lawrence Livermore National Laboratory under Contract No. W-7405-Eng-48.

$1 \quad$ J. J. Erpenbeck, Physics of Fluids 5 (10), 1181 (1962).

$2 \quad$ P.A. Isenberg, Astrophysical Journal 217 (2), 597 (1977).

3 D. Ryu and E. T. Vishniac, Astrophysical Journal 313 (2), 820 (1987). 
Figure 1: Experimental setup for laser-produced blast wave experiments. The 10JB. G. Elmegreen and D. M. Elmegreen, Astrophysical Journal 220 (3), 1051 (1978).

J. P. Ostriker and C. F. Mckee, Reviews of Modern Physics 60 (1), 1 (1988); G.

L. Welter, Astronomy and Astrophysics 105 (2), 237 (1982).

E. T. Vishniac, Astrophysical Journal 274 (1), 152 (1983).

D. Ryu and E. T. Vishniac, Astrophysical Journal 368 (2), 411 (1991).

E. Liang and K. Keilty, Astrophysical Journal 533 (2), 890 (2000).

G. Welter and J. Schmidburgk, Astrophysical Journal 245 (3), 927 (1981).

J. M. Blondin, E. B. Wright, K. J. Borkowski et al., Astrophysical Journal 500 (1), 342 (1998).

M. M. MacLow and M. L. Norman, Astrophysical Journal 407 (1), 207 (1993).

T. Ditmire, K. Shigemori, B. A. Remington et al., Astrophysical Journal

Supplement Series 127 (2), 299 (2000); K. Shigemori, T. Ditmire, B. A.

Remington et al., Astrophysical Journal 533 (2), L159 (2000); M. J. Edwards, A.

J. MacKinnon, J. Zweiback et al., Physical Review Letters 8708 (8), art. no. (2001).

J. Grun, J. Stamper, C. Manka et al., Physical Review Letters 66 (21), 2738 (1991).

; ; J. L. Rambo P. K. Rambo, Jr., G. R. Bennett, I.C. Smith, A.C. Erlandson, J.E. Murray, and J. Caird, OSA Trends in Optics and Photonocs, Conference on Lasers and Electro-Optics 73, 362 (2002).

J.A. Glaze, W.W. Simmons, and W.F. Hagen, Status of large neodymium glass lasers. (Soc. Photo-Optical Instrumentation Engrs, Palos Verdes Estates, CA, USA Reston, VA, USA, 1976).

Sir Geoffrey Taylor, Proc. Roy. Soc. A 201 (A), 159 (1950); L. Sedov, Prikl. Mat. Mekh. 10 (2), 241 (1946).

CALE Users Manual. (Lawrence Livermore National Laboratory, 1991). Inc. Cascade Applied Sciences, HYADES User's Guide. (Cascade Applied Sciences, Inc., Boulder, Co, 2004).

$1000 \mathrm{~J}, 1 \mathrm{~ns}$ drive beam enters one side of the chamber, illuminating a $0.5 \mathrm{~mm}$ solid target and creating a blast wave. A variable time later a probe laser is fired and comes in perpendicular to the drive beam, imaging the blast wave. Inset: Diagram of dark field telescope. Light that goes through the experiment 
undeflected gets blocked by a beam block at the focus of the imaging telescope. Deflected light shows up as a bright area on the CCD.

Figure 2: Images of blast waves in nitrogen at $25 \mathrm{~ns}, 100 \mathrm{~ns}$ and $300 \mathrm{~ns}$ after a $1000 \mathrm{~J}$ drive beam has hit a $0.5 \mathrm{~mm}$ nylon target. Bottom panel is a graph of the radius of the blast waves as a function of time. It shows that the blast waves follow a $t^{0.4}$ trajectory indicating that radiation losses do not play an important role in the hydrodynamics of the blast waves.

Figure 3: Images of blast waves in xenon at $50 \mathrm{~ns}, 200 \mathrm{~ns}$, and $800 \mathrm{~ns}$ after a $1000 \mathrm{~J}$ drive beam has hit a $0.5 \mathrm{~mm}$ nylon target. Bottom panel is a graph of the radius of the blast waves as a function of time. It shows that the blast waves follow a trajectory slower than $\mathrm{t}^{0.4}$ before $\sim 400 \mathrm{~ns}$, indicating significant energy losses due to radiation.

Figure 4: Zoomed in view of bump feature on blast wave 400ns after $1000 \mathrm{~J}$ laser pulse illuminated a $0.5 \mathrm{~mm}$ nylon target. Feature is caused by a portion of the blast wave front moving faster in the region heated by the drive laser's passage.

Figure 5: $9 \mathrm{~mm}$ spaced wire array in xenon gas 700ns after 1000J laser pulse illuminated $0.5 \mathrm{~mm}$ nylon target. The main blast wave is just passing the wire array. 
Additional blast waves created by radiation from the main blast wave have already formed around each wire in the array and are moving outward.

Figure 6: Evolution of perturbations on blast waves produced by a) $2 \mathrm{~mm}$, b) $4 \mathrm{~mm}$, and c) $6 \mathrm{~mm}$ spaced wire arrays. The perturbations damp out with time due to the high effective adiabatic index of the nitrogen gas.

Figure 7: Top panel is the coordinates of the edge of a blast wave moving past a $6 \mathrm{~mm}$ spaced wire array in polar coordinates. The bottom panel is the Fourier transform of the same shot. Highlighted is a peak at a wave number of $\sim 9$, which corresponds to the wave number of the induced perturbation of the wire array.

Figure 8: Amplitude of perturbations induced by a $6 \mathrm{~mm}$ spaced wire array on a blast wave traveling through nitrogen as a function of time. The measured decay rate can be compared to theoretical predictions.

Figure 9: Comparison of experimental results to theoretical predictions for decay rates as a function of wave number of perturbations for blast waves traveling through nitrogen. The experimental results match well to theoretical predictions for a gas with an adiabatic index of 1.4-1.5. 


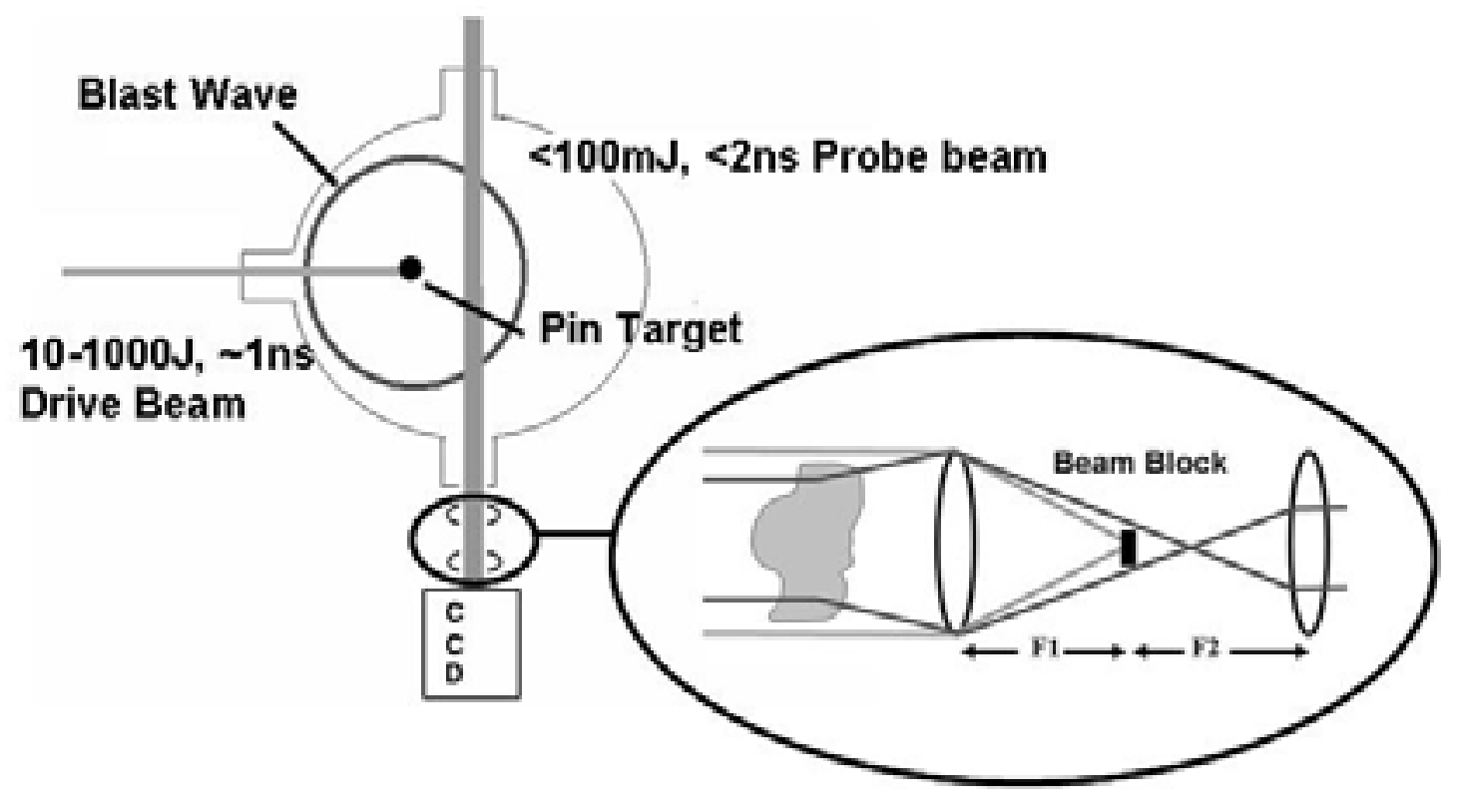



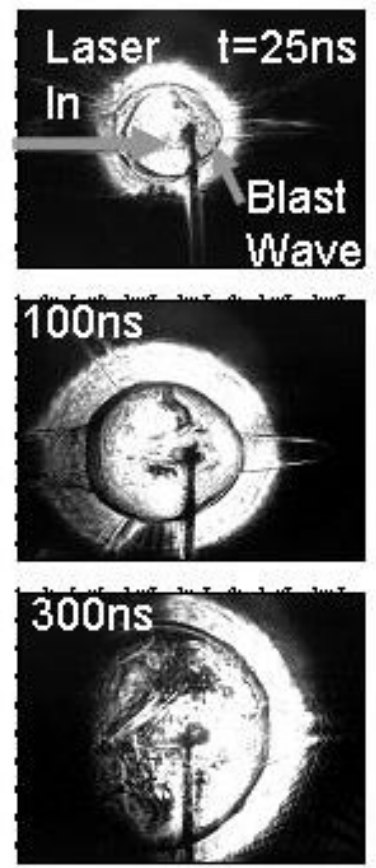

Nitrogen Shock Evolution

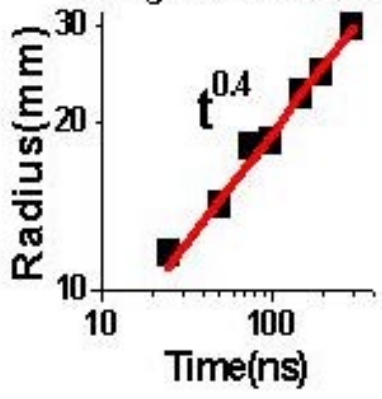




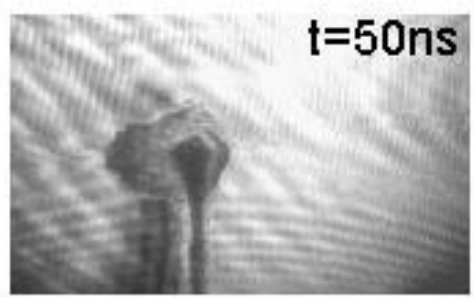

Blast Wave
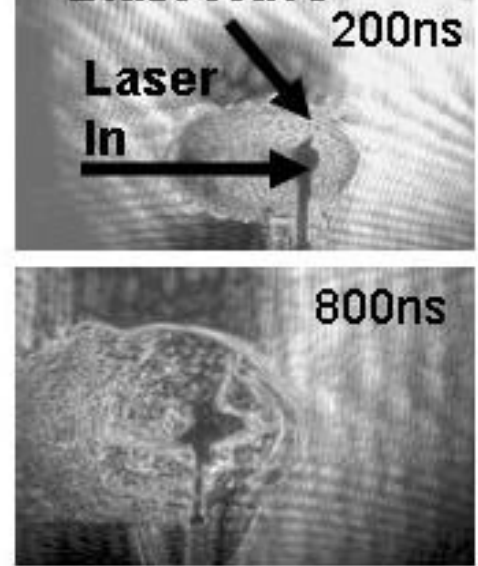

Xenon Shock Evolution

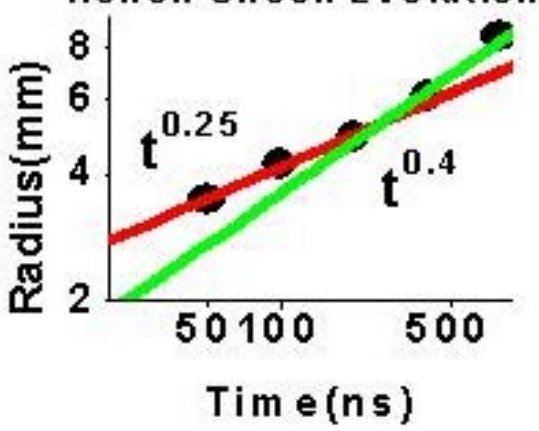




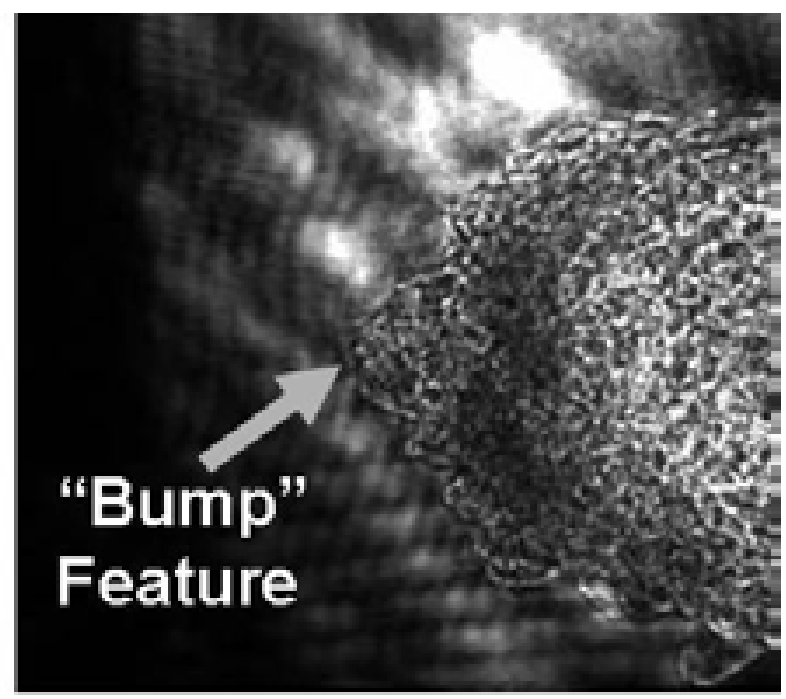




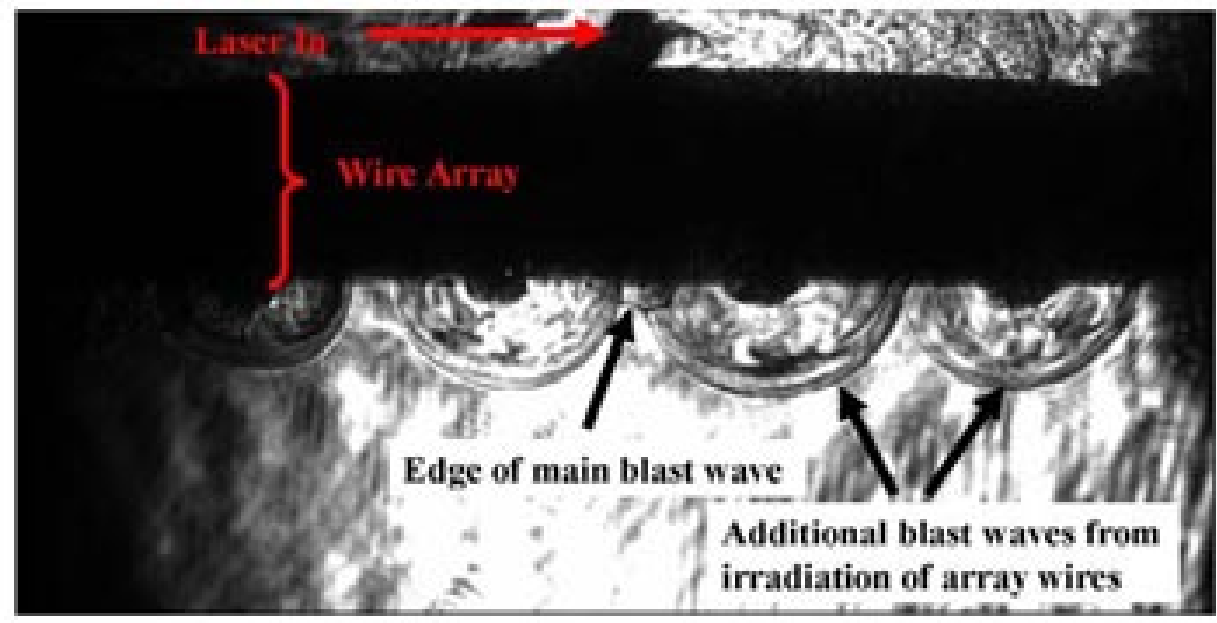




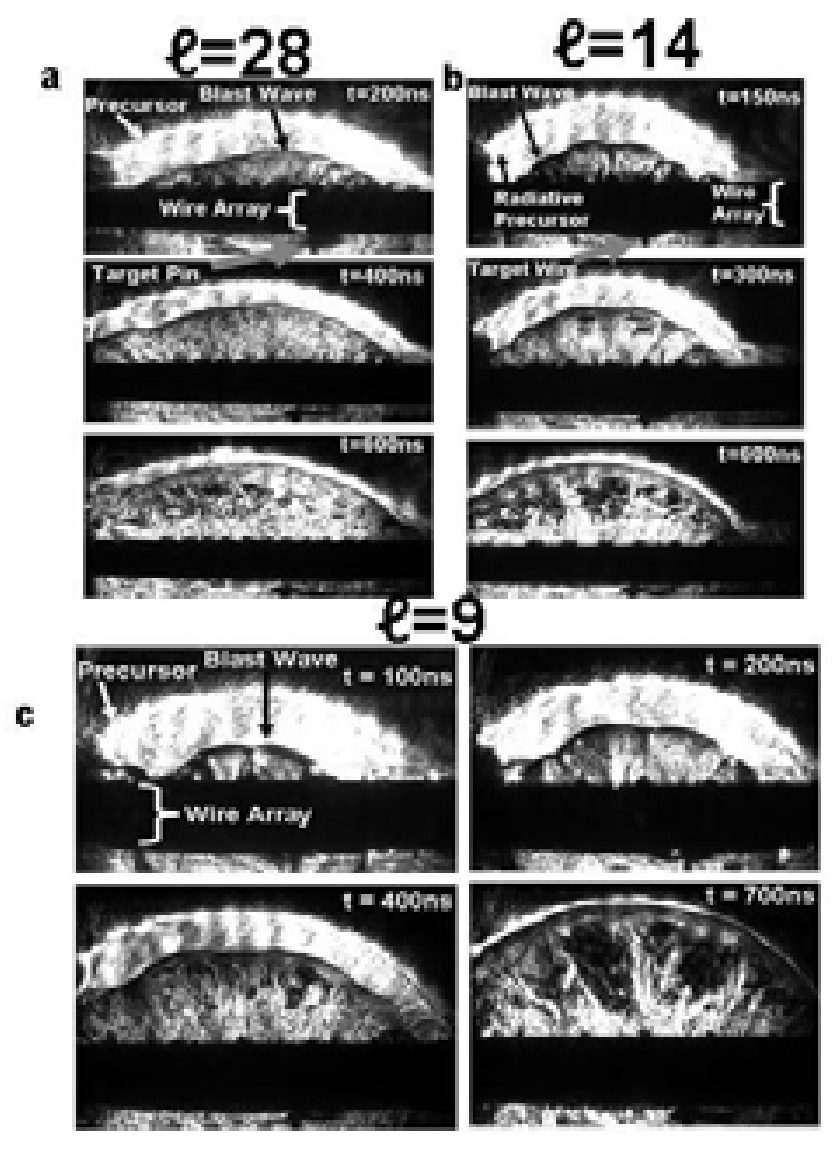




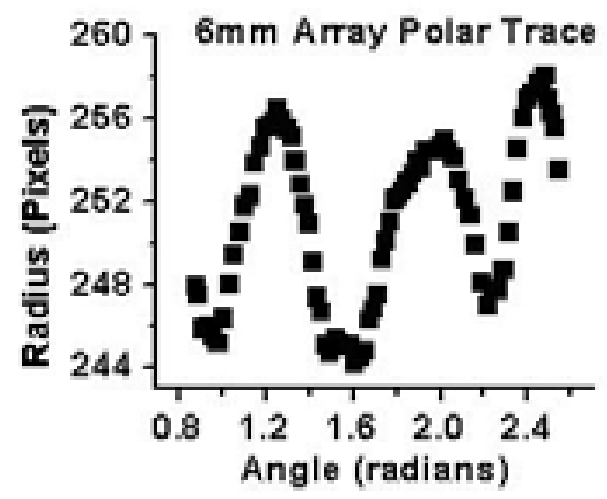

$6 \mathrm{~mm}$ Array Frequency Composition

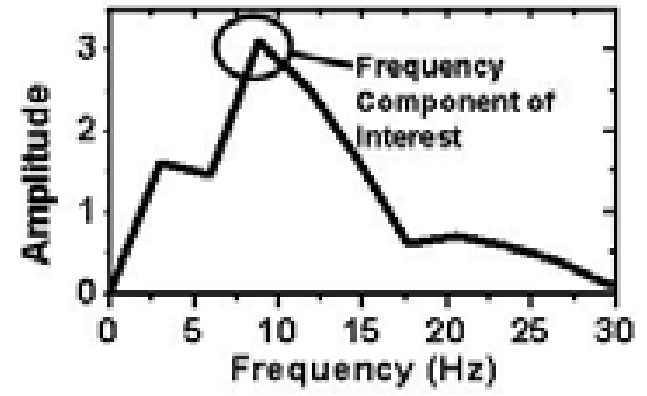




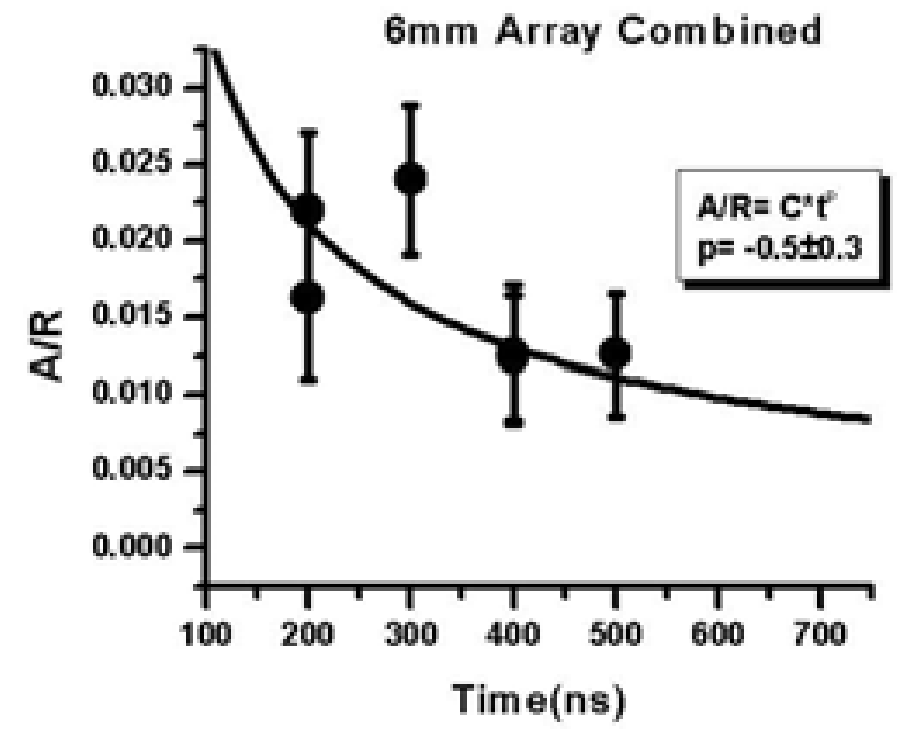




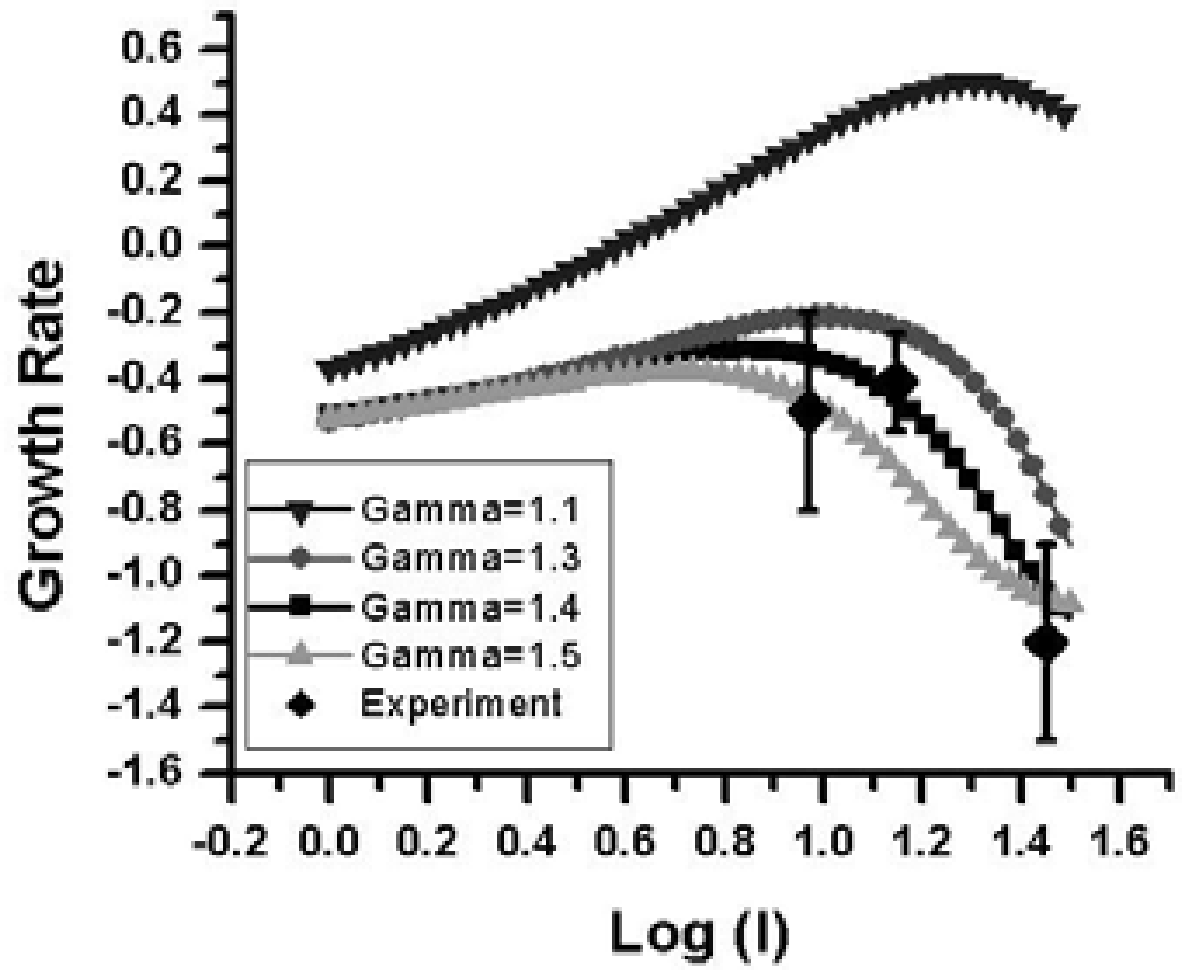

